\title{
Io and Its Plasma Environment
}

\author{
David J. Southwood, ${ }^{1,2}$ Margaret G. Kivelson, ${ }^{1}$ Raymond J. Walker, \\ AND JAMES A. SLAVIN'
}

\author{
Institute of Geophysics and Planetary Physics, University of California, Los Angeles, California 90024
}

\begin{abstract}
We describe and examine the interaction of Io with its plasma torus and the Jovian magnetic field in the context of several currently popular models. We address three specific matters. First, we discuss features implied by sub-Alfvenic flow which must be common to all models. Next, we examine the magnetic signature observed near Io by the Goddard Space Flight Center Voyager 1 magnetometer and point out that the preliminary estimate of $5 \times 10^{6} \mathrm{~A}$ current may be an overestimate. We obtain good fits with alternative current distributions which yield currents as small as $7 \times 10^{5} \mathrm{~A}$ through $\mathrm{Io}$. The best fits are obtained for an Alfven Mach number $\left(M_{A}\right)$ of 0.15 , but good fits are also found for $0.1<M_{A}<0.25$. Lastly, we point out the crucial role of charged particle data for probing the near Io interaction. We use published data from the low energy charged particle (LECP) detector to suggest that Io has an intrinsic magnetic field of magnitude comparable with earlier estimates. We make predictions which can test this picture as further data become available.
\end{abstract}

\section{INTRODUCTION}

Io, the innermost Galilean satellite of Jupiter has been recognized for nearly two decades as having a strong electromagnetic interaction with Jupiter itself; Bigg [1964] reported that Io modulated the intensity of radio waves in the decametric band emitted from Jupiter's vicinity. Goldreich and LyndenBell [1969] interpreted this as radiation associated with strong Birkeland (magnetic field aligned) currents flowing in the plasma between Io and Jupiter. The currents were believed to be set up because Io was a good conductor; the Jovian plasma on flux tubes intercepting Io would tend to move with Io and some source of energy would be needed to drag the flux tube feet through the Jovian ionosphere. Field-aligned currents transmit stress along the magnetic field and thus transfer momentum between Io and the Jovian ionosphere. A variety of scenarios for the Io-Jupiter interaction have been presented both before and since Goldreich and Lynden-Bell [1969] [Marshall and Libby, 1967; Webster et al., 1969; Gurnett, 1972; Shawhan, 1976; Goertz and Deift, 1973; Schatten and Ness, 1971], but the existence of a strong field-aligned current system which transmits stress between Io, the plasma in Io's vicinity, and the Jovian ionosphere has been a consistent feature of all models. Spacecraft exploration of the Jovian environment and of the magnetic shells in lo's vicinity in particular identified further important features of Io's interaction with the surrounding plasma. Radio occultation measurements by Pioneer 10 revealed that Io had an ionosphere [Kliore et al., 1975]. Webster et al. [1969] first suggested any currents carried by Io are likely to close through such a highly conducting region rather than through the solid part of the satellite. A second important plasma discovery was that Io was found to behave as a source of relatively low energy electrons (a few hundred keV energy) but as a sink at higher energies [McIlwain and Fillius, 1975; Thomsen, 1979].

The discovery of an ionosphere at Io raised some interesting questions. Cloutier et al. [1978] pointed out that there was a

\footnotetext{
' Also Department of Earth and Space Sciences, University of California, Los Angeles, California 90024.

${ }^{2}$ Also Blackett Laboratory, Imperial College, London, United Kingdom.

Copyright (C) 1980 by the American Geophysical Union.
}

considerable problem in understanding how the ionosphere was retained. An ionosphere is highly conducting and so the corotating Jovian magnetospheric plasma should induce substantial currents there. Cloutier et al. [1978] showed the $\mathbf{J} \times \mathbf{B}$ forces produced in the ionosphere should exceed the gravitational forces holding the material to Io and there should be a constant efflux of ions into the magnetosphere. They thus proposed a cometary model for Io. We sympathize with many of their views but emphasize that if Io has an intrinsic magnetic field it is substantially easier to understand how it can retain an ionospheric envelope that moves with it. We put forward this possibility in an earlier paper of ours [Kivelson et al., 1979] and look at it further here. The magnetic pressure of an intrinsic Io magnetic field can stand off the ambient Jovian field and plasma in a manner similar to what happens at Earth where the solar wind plasma and field are stood off to form the terrestrial magnetospheric cavity. Io differs in one very significant way with respect to the obstacles formed by planetary objects in the solar wind. Io is embedded in a subAlfvénic flow. This has several implications. Unlike the solar system magnetospheres we have directly sampled at Earth, Mercury, Jupiter, and Saturn or the ionospheric obstacle at Venus, Io has no shock in front of it. Concomitantly, pressure variations would be slight in its vicinity and flow perturbations are close to incompressible. This is true whether or not Io is magnetized and in either case similar flow perturbations are expected away from the immediate vicinity of the object.

Our earlier paper looked at Pioneer spacecraft data. Now the literature contains data from Voyager encounters with the Jovian environment. Voyager 1 passed about $20,000 \mathrm{~km}$ below Io and measured magnetic and particle flux changes directly attributable to Io's presence. Limited data are currently available but we analyze what has been published. We shall show that the orientation of Voyager's orbit relative to Io makes the energetic particle observations in Io's vicinity potentially a crucial means of distinguishing whether Io has an intrinsic magnetic field. As we show, currently published energetic particle data [Krimigis et al., 1979] seem to suggest the presence of a magnetic field at Io. Krimigis et al. [1979] had speculated thus. We show the putative field magnitude is comparable to that estimated by Kivelson et al. [1979]. Much of the 


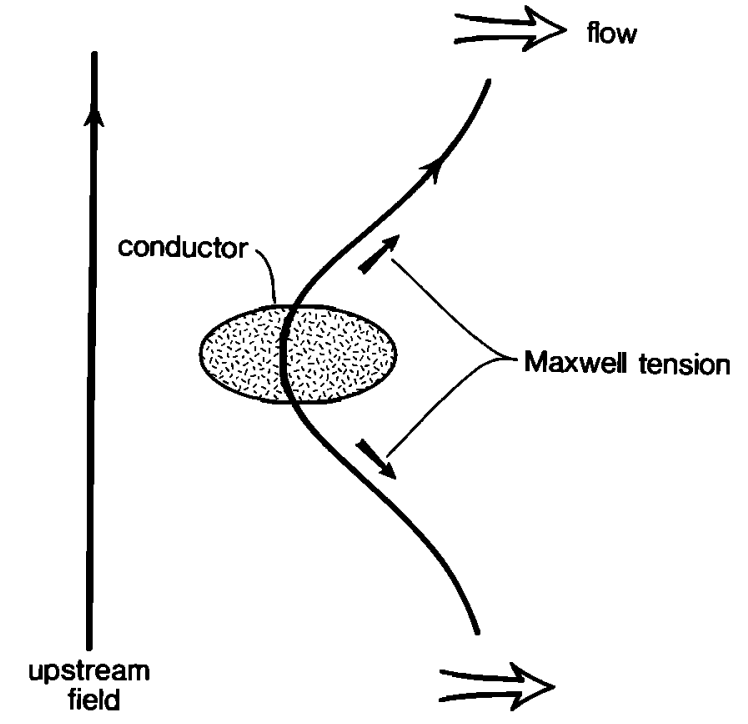

Fig. 1. Schematic illustration of the interaction of a conducting body with a magnetized flowing plasma.

additional information available cannot give evidence regarding the presence of an intrinsic field. We start by reviewing what happens if a conducting obstacle is present in a subAlfvénic plasma flow.

\section{Plasma flow in the Vicinity of a Finite CONDUCTIVITY OBSTACLE}

Although we do not expect a shock to form in Io's vicinity, flow diversion must take place. What occurs actually in the vicinity of an object such as Io depends on the precise physical nature of the object, but far from the object the flow pattern does not depend on details of the interaction. What matters is the effective conductance of the body as we describe next. Let us then consider what happens if an object that conducts electricity in an ohmic manner (i.e., that absorbs electromagnetic energy in so doing) is placed in a plasma flow.

The presence of a finite conducting object or region in a highly conducting flow produces a form of wake. The reason is straightforward. The electric field associated with the flowing plasma causes ohmic currents to flow in the conductor; flow energy is dissipated by Joule heating. The conducting regions absorb momentum from the flow in this process. Flow momentum is directed perpendicular to the magnetic field and is transmitted along the field by the appropriate component of the Maxwell stress tensor. The magnetic field perturbation that does this is perpendicular to the background field and is associated with currents directed along the magnetic field in the frame of the plasma. The Maxwell stress picture is a useful one and in Figure 1 we have schematically illustrated it. In MHD terms, outside the regions of finite conductivity the field is frozen into the flow. Near the conductor the electric field is reduced because the conductor can be polarized. The flow is thus reduced. The field gets 'caught up' in the conductor, bent and the resultant Maxwell tension is such as to slow the flow or to accelerate the conductor in the direction of the flow.

One need not picture the interaction this way. Many of the works referenced earlier, from Goldreich and Lynden-Bell on, talk in strictly electromagnetic terms. The motional emf induces currents in the conductor which flow out along the mag- netic field and then close across the background magnetic field in such a way that the associated $\mathbf{J} \times \mathbf{B}$ force slows the flow. The currents in the conductor naturally exert a force in the direction of the flow.

The interaction discussed above and illustrated in Figure 1 assumes that the skin depth of the conductor is large compared with its dimension. As we point out later this is not true in every possible model of the Io interaction. At this stage in our argument a failure of the magnetic field to penetrate fully makes little difference to our physical picture.

The field-aligned currents that communicate the stress between conductor and plasma flow in the moving plasma. A change in field-aligned current propagates along the field with the Alfvén velocity $B /\left(\mu_{0} \rho\right)^{1 / 2}$; the only MHD wave mode that carries field-aligned current is the Alfvén (or transverse) mode which is field guided [Dungey, 1968]. Because of this, in a flow which is sub-Alfvénic but whose Alfvén Mach number is finite, field-aligned currents from a perturbing object are carried off along $B$ by Alfvén waves in the plasma frame while in the object's frame, in which a steady state exists, the currents are carried back in the flow at the flow velocity while moving along B. Such a description was put forward by Drell et al. [1965] and the theory has recently been described and extended to cover nonlinearity and to apply to Io by Neubauer [1980]. The picture that emerges is illustrated in Figures 2 and 3.

Figure 2 illustrates a view which looks at Io at right angles to the Jovian field and flow direction. Although the current carrying region is shown concident with Io's cross section this is only schematic; the conducting regions of Io and its vicinity

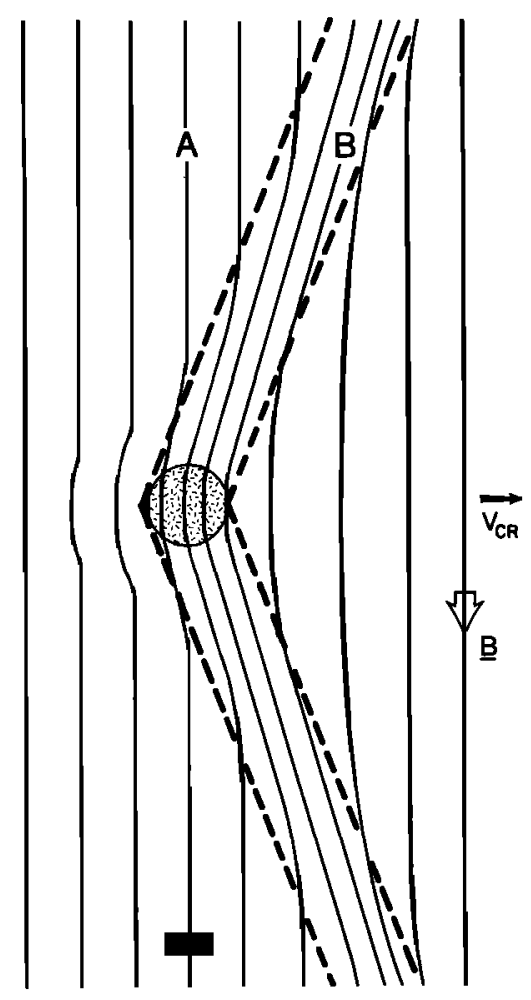

Fig. 2. Schematic illustration of the magnetic field in an azimuthal plane through the center of lo, assumed to be an unmagnetized conductor. Dashed lines represent boundaries of the Alfvén wings. The approximate locations of Voyager 1 as it crossed Io-associated field lines lie within the black rectangle. A and B label the positions of meridian planes through lo and through its wake, respectively. 
may have a far more complex cross section. From the sides of Io arise the Alfvén wings (Drell et al.'s [1965] term) which carry the currents between Io and the plasma. Sketched in the diagram are flux tubes which go through the central meridian of Io. Between the wings the field is most bent back but the perturbation does extend outside. So long as the flow is subAlfvénic and the plasma pressure small compared to field pressure, the actual field strength should be little perturbed by the interaction. The field line spacing in Figure 2 makes it clear that if the field strength is little perturbed, as we have argued, then field lines must bend out of the plane illustrated. Figure 3 illustrates this by showing a schematic of the field in two planes (A and B) perpendicular to the flow. The locations of these planes are indicated in Figure 2. Plane $A$ intersects the conductor and the bowing of the field is shown. Farther up the flux tubes communication with Io has not been established and the field is unperturbed. Further downstream in the flow, in plane $B$, perturbations due to Io have moved along the field as indicated. Because this plane does not intersect the conductor the perturbations are not present in the center. The conductor plucks the field lines as if they were stretched strings in a MHD picture of what occurs.

Various authors have used an Alfvén wave 'conductance' $\Sigma_{A}$ to parametrize the current in Alfvén waves set up by obstacles in flow (see, e.g., Maltsev et al. [1974], Mallinckrodt and Carlson [1978], and Neubauer [1980]). $\Sigma_{A}$ is given by

$$
\Sigma_{A}=\frac{1}{B}\left(\frac{\rho}{\mu_{0}}\right)^{1 / 2}
$$

If the Alfven wings are regarded as transmission lines linking with a cubelike conductor, the inverse of $\Sigma_{A}$ is the characteristic impédance of the line. We can define a voltage between the Alfvén wings. Say the voltage imposed across the wings by

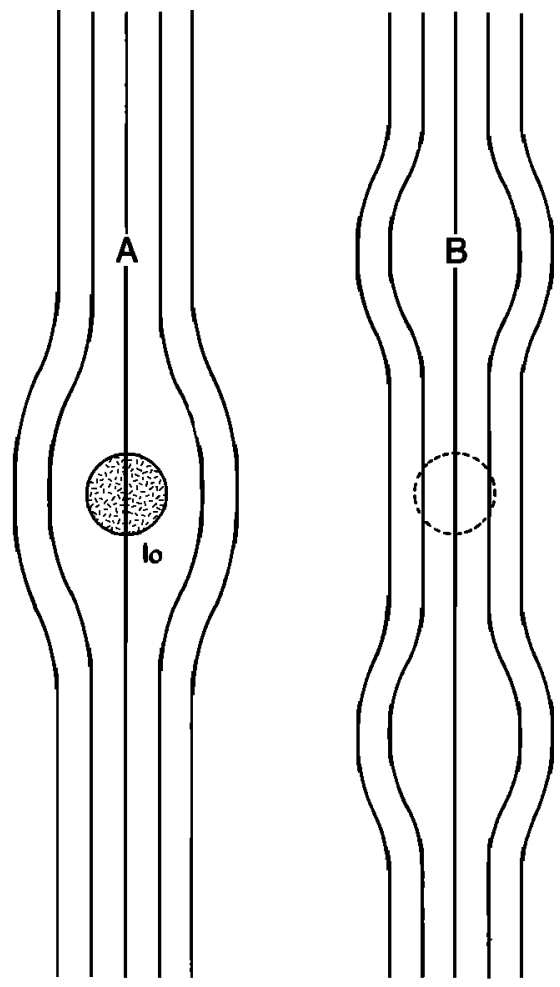

Fig. 3. Schematic illustration of the magnetic field in the meridian planes A (through Io) and B (through Io's wake) identified in Figure 2 .
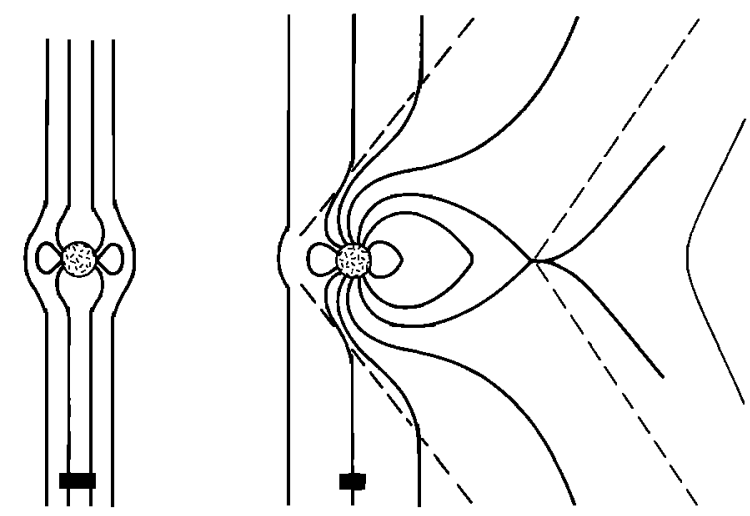

a) lo interaction viewed from upstream Projection of field lines on a plane through lo

b) lo interaction viewed radially inward towards Jupiter

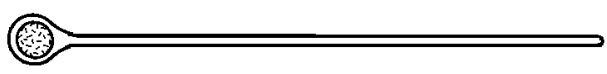

c) Region with field lines having one foot on lo projected onto equatorial plane

Fig. 4. Schematic illustration of magnetic fields near Io for the model of a reconnected magnetosphere. The black rectangles represent approximate locations of Voyager 1 as it crossed Io-associated field lines. (a) Field is viewed in a meridian plane through Io. (b) View in an azimuthal plane. Again Alfvén wings appear between upstream and downstream positions delimited by dashed lines. (c) An equatorial plane view of the region containing field lines which connect both with Io and with Jupiter.

the undisturbed flow is $V_{R}$. The Alfven waves reduce the flow over the object and say the wave voltage corresponding to this is $\Delta V$. The excess current produced at the conductor then is

$$
\Sigma\left(V_{R}-\Delta V\right)
$$

where $\Sigma$ is the conductance of the conductor. This excess is carried away by the wave and closes in the wave fronts, both above and below the conductor. In a uniform medium this implies

$$
\Sigma\left(V_{R}-\Delta V\right)=2 \Sigma_{A} \Delta V
$$

Immediately we can see that

$$
\frac{\Delta V}{V_{R}}=\frac{\Sigma}{\Sigma+2 \Sigma_{A}}
$$

This expression is easily interpretable. $\Sigma_{A}$ is proportional to the plasma mass density. If $\Sigma_{A}$ is large compared with $\Sigma$, comparatively little momentum and energy is absorbed from the flow by the conductor. This is reflected in the fact that $\Delta V \ll$ $V_{R}$; a relatively slight slowing of the flow by the conductor means that the adjustment in the electric field or potential made by the Alfvén wave to balance current is small. There would be little flow diversion and this circumstance would give rise to maximum plasma absorption. In the reverse situation where $\Sigma_{A}$ is much less than $\Sigma$, the Maxwell tension set up by the conductor's presence can more or less halt the flow; the momentum and flow energy of the plasma is relatively insignificant and the magnetic field is rooted into the conductor. The Alfvén urave signal almost switches off the applied signal, i.e., $\Delta V \sim V_{R}$. 


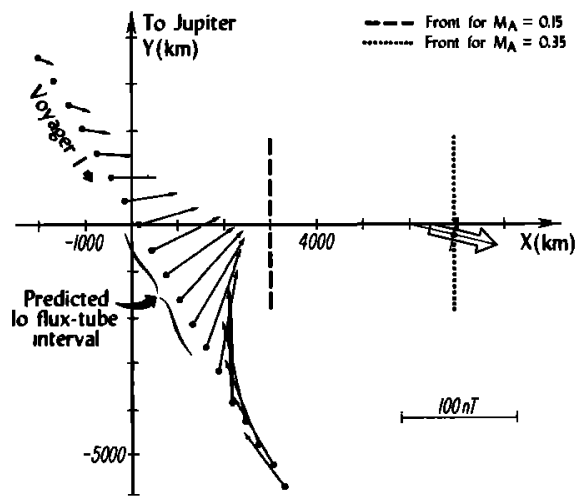

Fig. 5. Diagram adapted from Ness et al. [1979]. The magnetic perturbation vector in a plane parallel to the background magnetic field. The coordinate system is centered at and moving with Io. The corotation velocity lies in the $x-z$ plane. The position and direction of the best fit 2-D dipole moment [Ness et al., 1979] is shown by the arrow. Dotted lines indicate the expected upstream edge of the Io-associated Alfven wings for two different assumed Alfven Mach numbers.

The Alfvén waves traveling down the field eventually encounter the Jovian ionosphere where some form of partial reflection takes place. If the Jovian ionospheric height integrated conductivity, $\Sigma_{\boldsymbol{J}}$, exceeds $\boldsymbol{\Sigma}_{\boldsymbol{A}}$ the field lines cannot slip through the Jovian ionosphere and the reflected wave bends the field back further. After Io's passage the plasma will be brought back up to corotation also by a succession of Alfvén waves provided $\boldsymbol{\Sigma}$, is high enough. There is, however, some evidence that near Io plasma fails to actually corotate [see Bridge et al., 1979; Hill, 1979] and this imples $\Sigma_{J}$ is not high enough to freeze the field in totally.

\section{MAGNETOSPHERIC INTERACTION}

Let us now consider the specific features of a magnetospheric interaction between Io and its surrounding plasma. The critical value of Io's magnetic moment is about $5 \times 10^{22}$ $\mathrm{G} \mathrm{cm}^{3}\left(5 \times 10^{19} \mathrm{~A} \mathrm{~m}^{2}\right)$ for its field to start standing off the Jovian field and plasma. Kivelson et al. [1979] proposed a dipole moment of $6.5 \times 10^{22} \mathrm{G} \mathrm{cm}^{3}\left(6.5 \times 10^{19} \mathrm{~A} \mathrm{~m}^{2}\right)$. Now because Jupiter's dipole moment is tilted with respect to its rotation

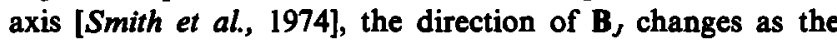
planet rotates. We shall however restrict ourselves to considering two extreme orientations in which Io's dipole is parallel or antiparallel to Jupiter's with recognition that neither could strictly apply over an entire orbit.

As Kivelson et al. [1979] describe, the Jovian plasma moving past Io will be diverted by the pressure of Io's field. Kivelson et al. [1979] pointed out also that the pressure balance was very largely between magnetic field pressures, Jupiter's and Io's; thermal and dynamic pressure are not important.

For the aligned dipole case, the fields on either side of the magnetopause are close to parallel and roughly equal in magnitude. Very strong currents would not form on the magnetopause. This is not true in the situation where Io and Jupiter's dipoles are antiparallel. Although field strengths would be equal on each side of the upstream magnetopause, the field directions are opposite and very large currents would be required to flow in a region of very weak magnetic field to achieve this configuration. In this circumstance a discharge phenomenon commonly called reconnection occurs [Dungey, 1975]. In the reconnection process energy is transferred to the plasma; the currents are dissipative. Reconnection couples the Jovian and Ionian fields. Some Jovian field lines enter Io's polar cap as Figure 4 indicates. Because the reconnection currents are dissipative a drag is exerted on the flow, and upstream and downstream of Io the configuration is markedly different. A tail should form extending downstream with fields close to antiparallel in northern and southern lobes, and with a weak field, strong current region in between where reconnection can occur.

Figure $4 a$ shows the interaction in a meridian plane through Io viewed from upstream in the flow. The view is analogous to that of Figure 3, although currents do not flow through Io but instead flow along the magnetopause in parallel with the system of field-aligned currents which close through the Ionian ionosphere as at Earth [Iijima and Potemra, 1976]. A cross-section viewed radially inward towards Jupiter is shown in Figure $4 b$. Dashed lines once again indicate the presence of the Alfven wings [Drell et al., 1965; Neubauer, 1980] which represent the boundaries of the region in which the principal perturbations are present. The field lines emerging from the polar caps are pulled downstream by the flowing plasma and fold over to form the magnetotail as at Earth. Flux conservation requires that the flux through one lobe of the tail equal the flux through the polar cap in which its field lines are embedded. If the polar cap field strength $B_{p c}$ is only slightly larger than the local Jovian field $B_{J}$ [Kivelson et al., 1979], the region of reconnected flux must be very narrow as illustrated schematically in Figure $4 c$. The length of the tail $\left(l_{T}\right)$ depends on $\epsilon$, the efficiency of reconnection, and $A$, the fraction of Io's surface area with field lines connecting to Jupiter [Kennel and Coroniti, 1975] and has been estimated as 32 $\boldsymbol{R}_{\mathrm{Io}}$ by Kivelson et al. [1979]. The width of the tail $\left(w_{T}\right)$ is obtained from

$$
B_{p c} A \pi R_{10}^{2}=B_{J} l_{T} w_{T}
$$

which with $A \leqslant 1$ gives $w_{T} \leq 0.1 R_{\mathrm{lo}}$.

From the tail reconnection region, previously noted, Alfver wings delineate the region in which transverse perturbations act to restore the Jovian field to its unperturbed state. The currents which flow in the Alfvén wings may vary as one moves downstream because the effective conductivity of the upstream current paths along the magnetopause and through the ionosphere may differ radically from the conductivity of the tail.

Either of the magnetospheres described would divert the bulk of the flowing plasma and allow Io to retain its ionosphere [Kliore et al., 1975] but only an open magnetosphere is consistent with the requirement that Io be strongly coupled with its plasma environment unless a strongly viscous interaction is invoked. In further discussion we consider only an open magnetosphere.

TABLE 1. The Angle, $\theta_{A}$, Between the Unperturbed Field Line Through Io and the Alfven Wings, and the Corresponding Horizontal Separation Between the Io Field Line and The Alfven Wings at a Distance $20,000 \mathrm{~km}$ Below Io, With Entries for a Range of Alfvén Mach Numbers, $M_{A}$

\begin{tabular}{ccc}
\hline$M_{A}$ & $\theta_{A}, \operatorname{deg}$ & $d, \mathrm{~km}$ \\
\hline 0.07 & 4.0 & 1400 \\
0.17 & 9.6 & 3400 \\
0.26 & 15 & 5200 \\
\hline
\end{tabular}




\section{Magnetic Field Perturbations in the Io Flux TUBE: OBSERVATIONS AND INTERPRETATION}

On March 5, 1979, the Voyager 1 spacecraft, outbound through the Jovian magnetosphere, passed beneath lo at a distance of 20,570 km [Stone and Lane, 1979]. Magnetic field perturbations and some energetic particle absorption were observed as the spacecraft crossed the nominal Io flux tube [Ness et al., 1979; Krimigis et al., 1979]. Ness et al. [1979], using data based on preliminary orbit attitude information, report that the field perturbations lie approximately in a plane transverse to the background Jovian field, $\mathbf{B}_{0}$. They introduce a coordinate system centered at and moving with Io with the $z$ axis aligned with the background field (positive northward) and the corotation flow direction in the $x-y$ plane. The projection of the magnetic perturbation vectors $\Delta B$ in the $x-y$ plane of this coordinate system and the Voyager 1 trajectory are shown in Figure 5, adapted from Ness et al. [1979]. The perturbation field magnitude is less than $100 \mathrm{nT}$ in a background field of $1900 \mathrm{nT}$, so the perturbed field lies within $3^{\circ}$ of the ambient Jovian field.

Ness et al. have modeled the observed perturbation field as the field of a two-dimensional (2-D) line dipole driven by twin currents, locally field aligned, and oppositely directed. Their best fit corresponds to a 2-D magnetic moment $\mathrm{m}|\mathrm{m}|=1.3 \times$ $10^{10} \mathrm{~A} \mathrm{~km}$ oriented $15^{\circ}$ outward from the $x$ axis, located at $x_{D}$ $=6950 \mathrm{~km}, y_{D}=-200 \mathrm{~km}$ as indicated by the large arrow in Figure 5.

The arguments presented in the previous section indicate that, regardless of the mechanism of plasma interaction, the currents linking Io flow along a pair of Alfvén wings. The Alfvén wings make an angle $\theta_{A}=\tan ^{-1} M_{A}$ with the field direction. Here, $M_{A}=\Delta v / v_{A}$ is the Alfvén Mach number, with $\Delta v$ the flow velocity of the Jovian magnetospheric plasma relative to Io and $v_{A}$ the Alfven velocity of the Jovian plasma. Voyager measurements indicate that near Io's flux tube the electron number density was $\sim 2000 \mathrm{~cm}^{-3}$ [Warwick et al., 1979], and the ionic mass per electron of the torus plasma was 16 proton masses [Bridge et al., 1979], indicating $v_{A}=230 \mathrm{~km} / \mathrm{s}$. If the plasma near Io was corotating, $\Delta v \simeq 60$ $\mathrm{km} / \mathrm{s}$ and $M_{A}=0.26$. On the other hand, the characteristic thermal velocity of the doubly ionized sulfur ions in the Io

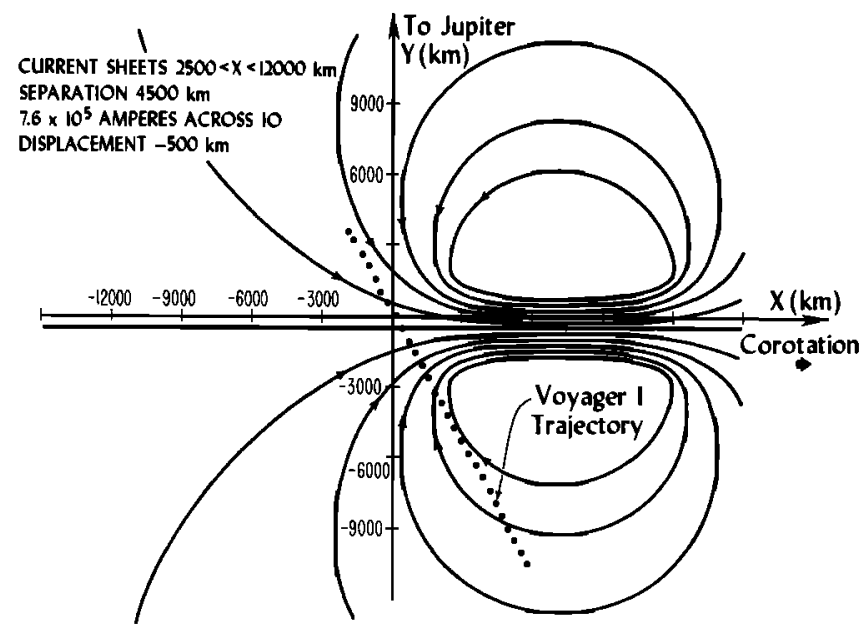

Fig. 6. Field lines predicted from model current sheets centered $500 \mathrm{~km}$ radially outward from the $x-z$ plane. The sheet lengths, separations and the current across one Io diameter are given in the figure.
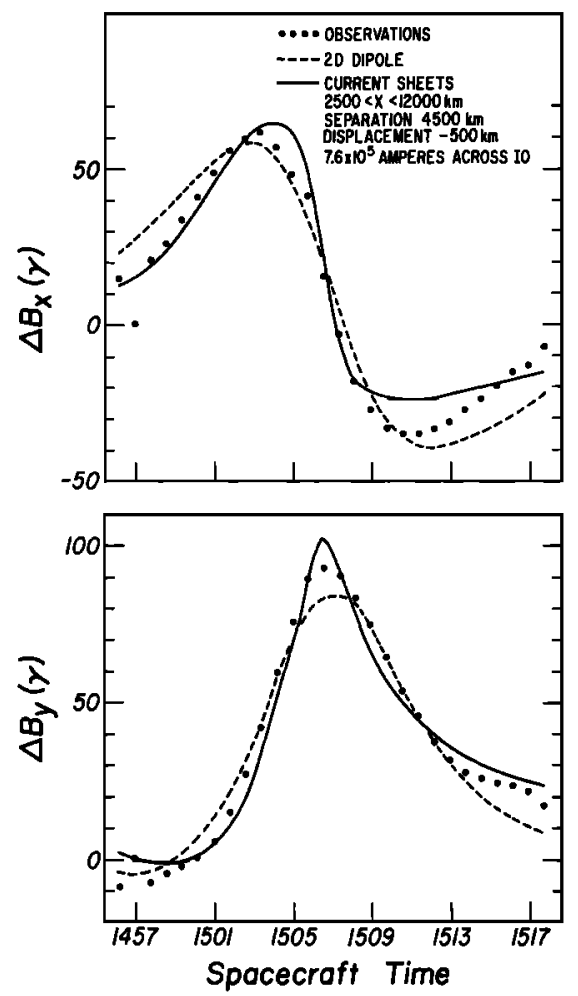

Fig. 7. Observed perturbation magnetic field components (dots) $\Delta B x$ and $\Delta B y$ and best fit magnetic field for twin oppositely directed currents (dashed curve) (adapted from Ness et al. [1979]). Solid curves are perturbation field components obtained for the current sheets illustrated in Figure 6.

torus is $\sim 16 \mathrm{~km} / \mathrm{s}$ [Bridge et al., 1979]; this velocity is thought to correspond to the relative flow velocity for plasma in the vicinity of Io where the sulfur atoms leaving Io are ionized (G. Siscoe, personal communication, 1979). The reduced flow speed is expected in regions where a source of plasma exists [Hill, 1979]. If the ionization occurs upstream of Io, the effective Mach number will be reduced. For a $16-\mathrm{km} / \mathrm{s}$ relative flow velocity, one finds $M_{A}=0.07$. This lower limit constrains the Mach number to the range between 0.07 and 0.26 .

The Mach number determines the angle $\theta_{A}$, which in turn indicates the horizontal distance at the latitude of the Voyager 1 spacecraft between an unperturbed field line through Io and the region in which Io-associated currents flow; this distance is denoted ' $d$.' Table 1 gives values of $\theta_{A}$ and $d$ for $M_{A}$ in the range of physical interest. For the probable small values of $M_{A}$, the perturbation currents should flow at a distance of less than one Io diameter $(3600 \mathrm{~km})$ from the spacecraft as it traverses the nominal flux tube. This distance is significantly less than the $7000 \mathrm{~km}$ separation between Voyager 1 and the dipole current of the Ness et al. [1979] model, a distance which would correspond to a Mach number of 0.35 .

If the Io-associated currents flow within $2 R_{\text {Io }}$ of the spacecraft trajectory, the spatial distribution of the currents in the $x-y$ plane cannot be ignored, and a model current system with finite horizontal extent should represent the currents better than a 2-D dipole model. The finite spread of the currents can be modeled by allowing field-aligned antiparallel currents to flow along plates of $x$ dimension $L$ and $y$ separation $2 a$. For small $M_{A}$ such sheet currents would flow in the region between the upstream and downstream Alfvén wings of Figures 


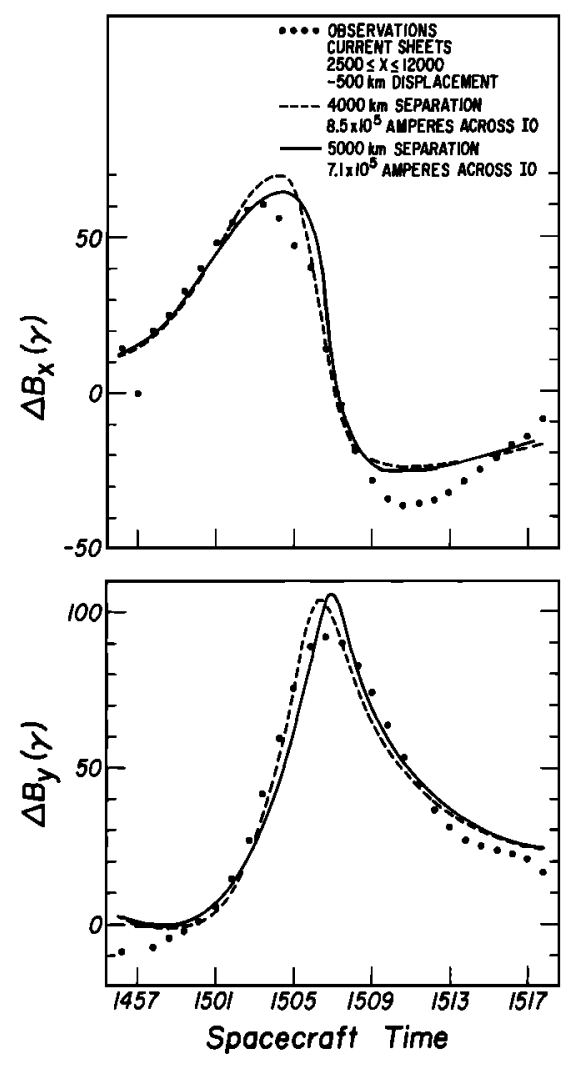

Fig. 8. Comparisons of measured (dots) and model perturbation fields predicted from current sheet model for two different sheet separations.

2 or 4 . The $x$ position of the upstream edge of the sheet currents is set by the assumed $M_{A}$. In the simplest model, only three additional parameters need be specified: $L, 2 a$, and the sheet current density $j$.

Figure 6 illustrates the field lines obtained for current sheets $9500 \mathrm{~km}$ long, with the upstream edge at $2500 \mathrm{~km}$ from the origin of the coordinate system. The track of the Voyager 1 spacecraft lies along the dotted path. In Figure 7 the measured perturbation field components along the Voyager 1 trajectory (dots) are compared with the predicted values of the field produced by a current sheet (solid curve) with the parameters of Figure 6. Also plotted is the perturbation field arising from the model 2-D dipole source of Ness et al. (dashed curves). A constant field was added to the $y$ component to improve the fit as the reference level of field is arbitrary in this presentation. For the sheet current model the addition of $20 \mathrm{nT}$ gave the best fit and for the dipole model the addition was $10 \mathrm{nT}$. The addition of a constant to the $x$ component did not improve the fit for either model.

Success in obtaining acceptable fits to the limited perturbation magnetic field data does not necessarily imply that the model currents reflect the physical situation. This is apparent from Figure 7, which includes very similar calculated curves corresponding to two significantly different model current systems. Nonetheless, the sheet current calculation can tell us several things about the physical current system. First, it shows that the inferred current flowing through Io is model dependent. In particular, the current across $2 R_{\text {Io }}$ is 6 times smaller $\left(7.6 \times 10^{5} \mathrm{~A}\right)$ for the sheet current model than for the 2-D dipole model $\left(4.8 \times 10^{6} \mathrm{~A}\right)$. Indeed, the smaller the spatial separation between the distributed currents and the space- craft trajectory, the smaller the current required. Other models we have tested give good fits to the data and reduce the current through Io to as little as $7 \times 10^{5} \mathrm{~A}$. The magnitude of the current across the flux tube must be known in order to calculate magnetic torques on Io (S. Peale, personal communication, 1979) or to evaluate the Joule heating of Io and the Io plasma torus [Ness et al., 1979], so it is important to recognize the range of values compatible with the data.

Second, the sheet current model is a zeroth order approximation to the current structure anticipated on physical arguments, and its parameters represent characteristic scale lengths. In Figures 8 to 11, we show the modifications produced by systematically changing model parameters. Figure 8 shows the response of the peak perturbation field components as the sheet separation is changed. Figure 9 shows that the current density and the variation of the perturbation field near its maximum changes as the distance to the inner edge of the sheet increases. Figure 10 shows the result of radial displacement of the mid-plane of the current sheets. Figure 11 shows the minor modifications which result from increasing the length of the current sheet by almost a factor of 3 . For each parameter set, the current has been adjusted to minimize the deviation between observations and predictions. Our search over parameter space has led us to conclude that the sheet separation must be of the order of Io's diameter and that the length of the sheets cannot be less than one Io diameter to obtain a reasonable fit. The fact that the measured perturbations would not be very different for long or short current sheets means that the magnetometer measurements cannot be used to distinguish between the short current sheets expected for the unmagnetized conductor (Figure 2) and the long current sheets anticipated for a reconnected magnetosphere (Fig-

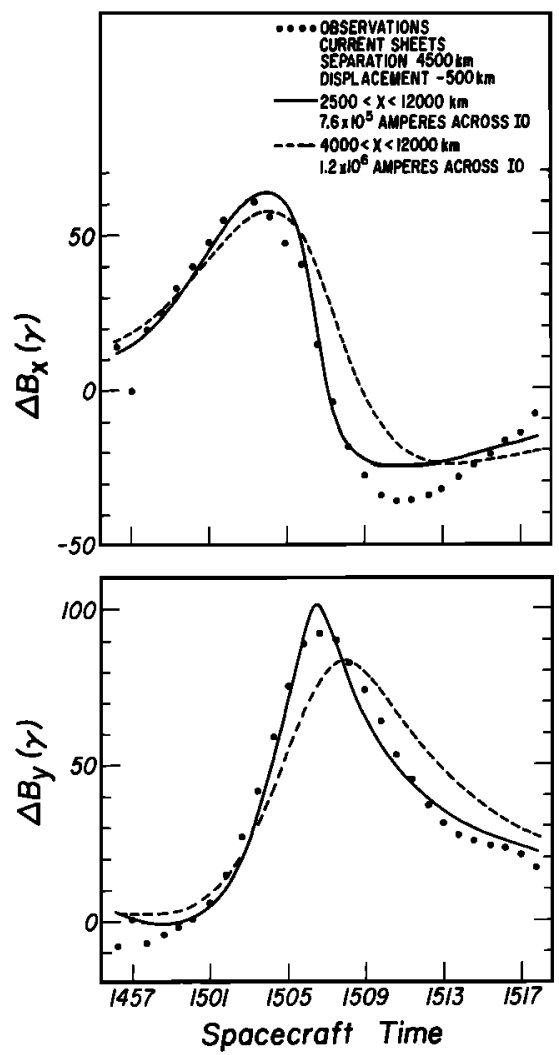

Fig. 9. Same as Figure 8 for two different positions of the upstream edge of the sheet. 
ure 4). The fact that both parameters, length and separation, of the plates as obtained from fits to the field data correspond to the expected dimensions of an Io interaction region encourages us to believe that the model we have used reflects meaningfully the parameters of the currents actually flowing in Io's vicinity.

Third, the location of the upstream edge of the sheet is intimately related to the plasma conditions at Io. For reasonable separation distances, good fits were obtained for $M_{A}$ between 0.1 and 0.25 . The fits we obtain suggest that the currents were quite close to the Voyager 1 trajectory and could be consistent with $M_{A}$ as low as 0.1 , and thus with very much reduced plasma flow velocity in Io's vicinity.

\section{Particle Fluxes in the Io Flux Tube: OBSERVATIONS AND INTERPRETATIONS}

We now turn to the question of whether Voyager 1 encountered the Io flux tube (i.e., whether it crossed any field lines which passed through Io). Figure 2 shows the schematic geometry of the field coupling Voyager and Io. The black rectangle contains the path of Voyager as it passed Io-associated field lines. The Mach number has been assumed unrealistically large $\left(M_{A} \simeq 0.4\right)$ so that the perturbed region can be indicated clearly. The currents are confined to a region upstream of Io; but the field line through Voyager 1, only slightly perturbed in the region traversed by the spacecraft, and somewhat more perturbed near Io, does pass through both bodies. We believe that the Voyager 1 particle data initially reported [Krimigis et al., 1979] are consistent with the suggested geometry. We take issue with the suggestions [Krimigis et al., 1979; Ness et al., 1979] that Voyager did not pass
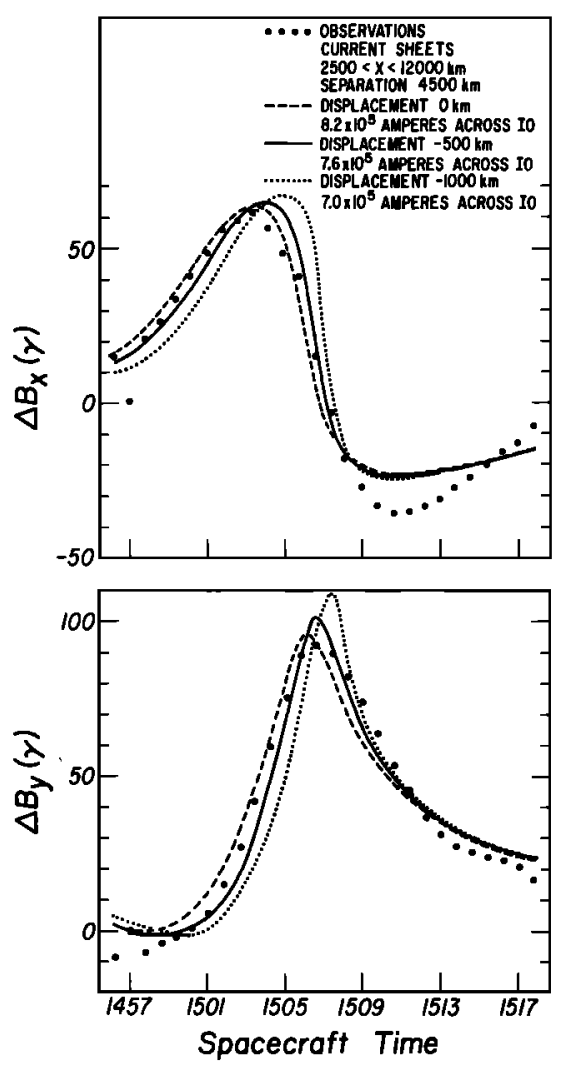

Fig. 10. Same as Figure 8 for three different radial displacements of the center of the two current sheets relative to the $x$ axis of Figure 5.
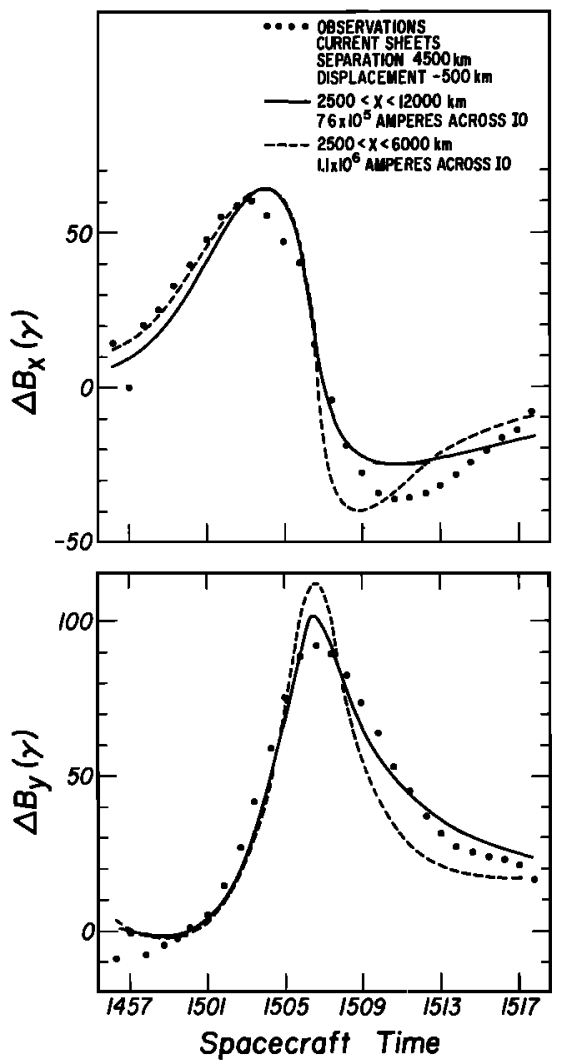

Fig. 11. Same as Figure 8 for two different sheet lengths.

through the Io flux tube although we do agree that it did not pass through the region in which the Io-related currents flowed.

The energetic particle fluxes reported by Krimigis et al. [1979] from measurements made as the Voyager 1 spacecraft crossed the Io flux tube are shown in Figure 12. Small fluctuations are present in the fluxes of ions, but one may question whether any of the perturbations are clearly associated with the Io flux tube (i.e., the region of field lines passing through Io). The $\geq 10 \mathrm{MeV}$ electron flux, on the other hand, decreases by $35 \%$ as the Io flux tube is crossed, and returns to high levels immediately thereafter. This dropout seems to be clearly an Io effect.

The flux signatures observed are consistent with the assumption that Voyager 1 crossed the Io flux tube if the guiding center motion of the particles is considered. In Figure 13, the guiding center motion is shown schematically between Io's and Voyager's latitudes for particles mirroring at the spacecraft. Particles whose average parallel velocity is not large compared with their azimuthal drift velocity reach Voyager 1 having crossed Io's latitude upstream of Io; such particles can at most record the small effects produced upstream of Io by fast mode waves. Information on direct interaction with Io must be sought by examining the flux of particles whose average parallel velocity is large with respect to their azimuthal drift velocity.

To determine quantitatively which particle fluxes should show effects produced by Io, we have plotted the azimuthal distance travelled in a quarter bounce time as a particle moves from the equator to its mirror point as a function of energy for electrons and protons in Figure 14. We have assumed full corotation and have added magnetic drift effects. If during the nominal flux tube crossing Voyager 1 was indeed on field lines 


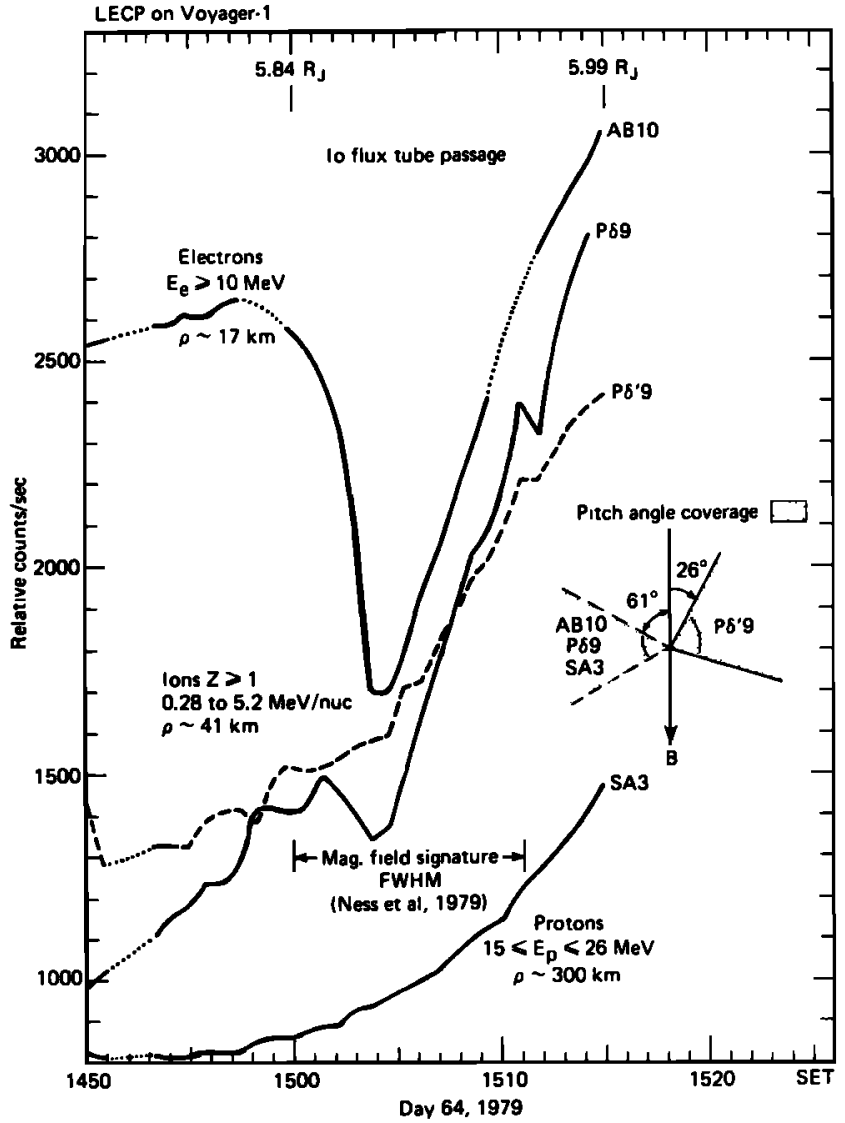

Fig. 12. Measurements of fluxes of electrons and ions from the Voyager 1 low energy charged particle detector, reproduced from $\mathrm{Kri}$ migis et al. [1979].

threading Io, only particles whose azimuthal drift during a quarter bounce period was $\leqslant 1 R_{\mathrm{Io}}=1800 \mathrm{~km}$ could have encountered Io en route to the spacecraft. This means protons with $E \geqslant 1 \mathrm{MeV}$ and electrons with $E_{e} \geqslant 0.3 \mathrm{keV}$ could have interacted with Io before reaching the detectors. If the flow velocity is less than the corotation velocity, the interaction energy decreases for electrons but remains essentially unchanged for protons (for $M_{A} \simeq 0.07, E_{p} \simeq 700 \mathrm{keV}$ ). Finite gyroradius effects may be important for the high-energy proton detector ( $15 \leq E_{p} \leq 26 \mathrm{MeV}$ ), especially if the field lines at Io are confined to the ionosphere, so we will limit further discussion of measured fluxes to the other three traces of Figure 12. Then, as we rationalize on the basis of guiding center motion, only the energetic electrons $(\geq 10 \mathrm{MeV}$ ) evidence a clear Io effect. Io's effects on protons of a few hundred $\mathrm{keV}$ would have been detected if the spacecraft trajectory had crossed Io's orbit either somewhat downstream of Io or closer to its latitude.

In using trajectory analysis to delimit the energies at which an Io signature should appear in measured particle fluxes, we have not specified the nature of the particle-moon interaction. The effect we have discussed is purely geometrical for a specified flow velocity. Indeed, the minimum energy at which an Io signature is observed for different particle species should give independent evidence for the actual plasma flow velocity in the vicinity of Io.

For a range of energies near the minimum energy at which an Io signature is possible, the flux dropout may be sensitive to pitch angle. The effect is illustrated in Figure 15, where por- tions of the guiding center trajectories for fixed energy protons of different pitch angles are shown schematically. After crossing Io's latitude, particles of $\$ 90^{\circ}$ pitch angle may travel directly to the spacecraft while moving through a limited azimuthal distance. At other pitch angles, particles must travel to their mirror points and back up to the spacecraft experiencing azimuthal displacement throughout. The case illustrated in Figure 15 shows that the flux of particles mirroring below the spacecraft may be unaffected by Io at a time when the flux of locally mirroring particles is modified by an Io interaction. Once again the effect depends on the field geometry and the plasma flow velocity, and should be useful in establishing the plasma flow velocity near Io.

The flux of 0.28 to $5.2 \mathrm{MeV}$ nucleon ions measured by one of the two ion detectors (see Figure 12) dropped slightly near the Io flux tube crossing, but no analogous dip was observed in the second detector with the same energy response. The detectors measured fluxes at different pitch angles (see insert, Figure 12). The flux decrease occurred for particles mirroring near the spacecraft, and was absent in the fluxes which included particles mirroring well below the spacecraft. The pitch angle dependence of the small Io-associated ion flux perturbation is qualitatively consistent with the arguments presented in the discussion of Figure 15, but more complete pitch angle data is needed to carry this argument further.

\section{EVIDENCE FOR AN INTRINSIC MAGNETIC FIELD AT Io}

Having argued that only the energetic electron channel of Figure 12 should have registered effects of interaction with Io, we ask what the fluxes of these $\geq 10 \mathrm{MeV}$ electrons can reveal about the nature of that interaction. Suppose, for example, that Io is a conductor whose skin depth is at least comparable with its radius. Then energetic electrons on field lines through Io will be lost through collisions with the moon or its atmosphere. This should produce a complete dropout of energetic electron flux where the Voyager 1 trajectory crosses field lines threading Io. On field lines which do not thread Io, there should be virtually no losses.

Figure 12 shows that the $\geq 10 \mathrm{MeV}$ electrons did not dis-

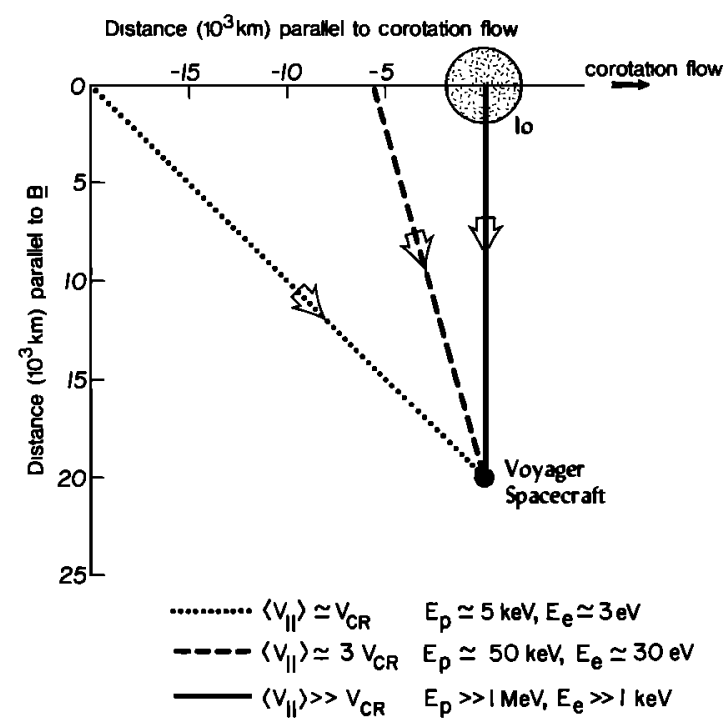

Fig. 13. Schematic of guiding center trajectories of charged particles of different energy and $90^{\circ}$ pitch angle at the position of Voyager l. 
appear in the Io flux tube, Instead, their flux decreased by about 35\%. Diffusion cannot refill the flux tube fast enough to explain why the flux does not vanish. If we use the largest diffusion coefficient for $10 \mathrm{MeV}$ electrons provided by Simpson et al. [1974] and by Mogro-Campero and Fillius [1976] $\left(D_{L L}=\right.$ $10^{-6} \mathrm{~s}^{-1}$ at Io), we obtain a lower limit for the characteristic diffusion time scale, $\tau_{D}$. With $\tau_{D}=\left\langle\Delta L^{2}\right\rangle / D_{L L}$ and $\left\langle\Delta L^{2}\right\rangle=$ $\left(R_{\mathrm{Io}} / R_{J}\right)^{2}$ we find $\tau_{D}=660 \mathrm{~s}$. This diffusion time scale exceeds the electron half-bounce $(0.35 \mathrm{~s})$ by such a large factor that losses strongly dominate diffusion sources. The losses dominate diffusion even in the limit of Bohm diffusion ( $\left.\tau_{D} \sim 10 \mathrm{~s}\right)$. The argument is little changed if the Jovian field lines are confined to the ionosphere of Io rather than its interior. For this case, the energetic electrons could not reach Io's surface, but losses would occur in the Ionian atmosphere.

If Io has its own magnetic field, particles incident from the Jovian magnetosphere are not necessarily lost. For example, if Io has a dipole field with dipole moment roughly antiparallel to Jupiter's (see section 3), large pitch angle particles approaching Io may mirror in Io's polar cap field before reaching its atmosphere or its surface. This consequence of an intrinsic Ionian field was discussed by Kivelson et al. [1979] who noted that their suggested dipole moment $\left(6.5 \times 10^{19} \mathrm{~A} \mathrm{~m}^{2}\right)$ should cause particles with pitch angles roughly between $70^{\circ}$ and $110^{\circ}$ to mirror in Io's polar caps. If a dipole field model is used to trace the pitch angles from Io's latitude to Voyager's, the corresponding range of pitch angles is $75^{\circ}$ to $105^{\circ}$. Krimigis et al. [1979] measured electrons with a range of pitch angles centered between $61^{\circ}$ and $119^{\circ}$ as indicated in the inset, Figure 12. If the unperturbed electron flux was isotropic, half the flux in the pitch angle range measured would mirror in Io's polar cap, and only half the flux would be lost, which is roughly in agreement with observations.

Measurements of the pitch angle distributions inside and outside of the Io flux tube could in principle determine whether the losses depend on pitch angle in the manner described. Furthermore, the strength of the Ionian magnetic moment can be obtained quite accurately from the pitch angle distribution of the energetic electron flux in the Io flux tube.

\section{Conclusions and Predictions}

We have argued that the small perturbation magnetic fields measured during the Voyager 1 traversal of the Io flux tube

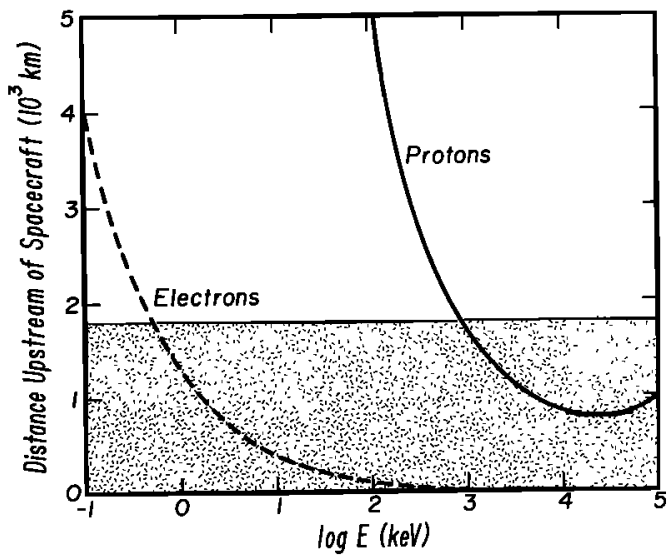

Fig. 14. Azimuthal distance traveled by electrons and protons in the time between their arrival at the Voyager 1 spacecraft and their previous crossing of the magnetic equator, assuming $90^{\circ}$ pitch angle at Voyager 1 , plotted as a function of particle energy. The shaded region corresponds to one $R_{10}$ in the azimuthal direction.

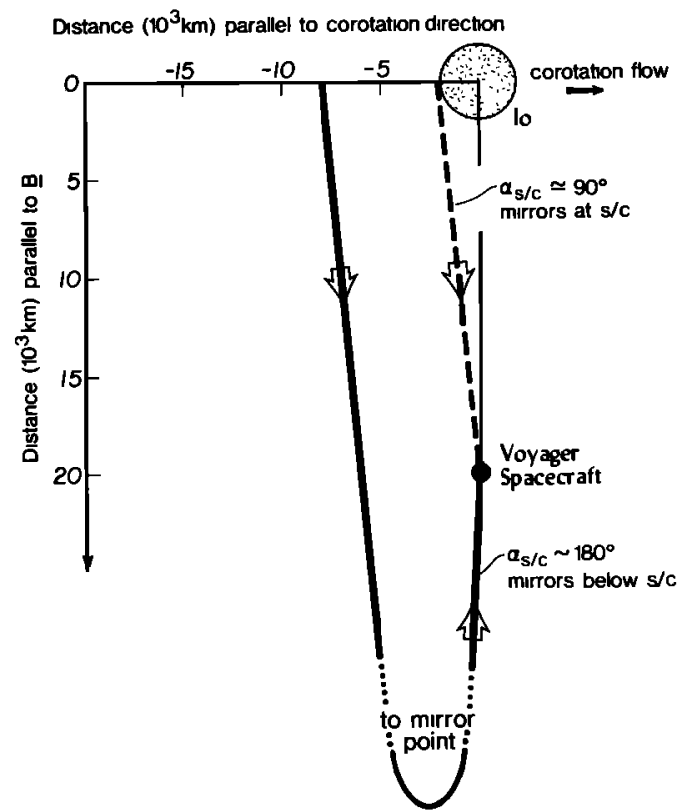

Fig. 15. Schematic guiding center trajectories for energetic protons of the same energy but different pitch angles at Voyager 1. $E_{p} \simeq$ $500 \mathrm{keV}$.

are consistent with expected forms of Io's interaction with the Jovian plasma. Using a model current system based on the theory of Alfven wave propagation, we have estimated the current flowing through Io. Our lowest estimate of $7.0 \times 10^{5} \mathrm{~A}$ suggests that the current could be almost a factor of 7 less than Ness et al.'s [1979] initial estimate.

We have pointed out that the particle fluxes described by Krimigis et al. [1979] are consistent with the assumption that Voyager 1 crossed field lines threading Io because a large Ioassociated flux decrease characterized the flux measured by the energetic electron detector, the only detector counting particles whose guiding center trajectories could have crossed Io en route to the spacecraft. For the other detectors, the absence of clear signatures of lo shows only that most of the measured particles followed guiding center trajectories which did not pass near Io.

The above interpretation allows us to predict the Io signature in other energy channels. For each particle species, fluxes should show an lo dropout above a minimum energy. If the Jovian plasma is corotating this minimum energy is approximately $1 \mathrm{MeV}$ for protons, and a few tenths of a $\mathrm{keV}$ for electrons. (Above about $10 \mathrm{MeV}$, finite gyroradius effects may be important for protons, and guiding center arguments fail.) The minimum energy at which flux dropouts are present should be pitch angle dependent with the minimum energy increasing monotonically with increase of particle pitch angle from $0^{\circ}$ to $180^{\circ}$.

Measurements of the pitch angle distributions of the particles within the region of decreased flux give the only direct clue regarding the nature of the lo interaction. The data available at this time support the suggestion that Io is magnetized. In that case, the pitch angle distribution of energetic electrons near the center of the flux decrease should be peaked near $90^{\circ}$ pitch angle, and should fall off sharply at critical pitch angles symmetric about $90^{\circ}$, unless strong wave-induced pitch angle diffusion is present. The high time resolution wave spectrum in the Io flux tube has not yet been fully described (but see Scarf et al. [1979]). 
If further analysis of particle data confirms the existence of a magnetic interaction at Io, it may be possible to establish whether Io has a large scale 'planetary' magnetic field, or whether particle mirroring is produced by crustal magnetization. The analysis of energetic particle pitch angle distributions has proved useful in the somewhat analogous study of the scale of magnetic anomalies on the Earth's moon [Lin et al., 1975].

Acknowledgments. We would like to acknowledge the assistance of the referees in clarifying aspects of the data interpretation. This work was supported by the National Aeronautics and Space Administration under contracts NAS 9-55232, NSG 7295; and NGR 05-007271.

\section{REFERENCES}

Bigg, E. K., Influence of the satellite Io on Jupiter's decametric emissions, Nature, 203, 1008, 1964.

Bridge, H. S., J. W. Belcher, A. J. Lazarus, J. D. Sullivan, R. L. McNutt, F. Bagenal, J. D. Scudder, E. C. Sittler, G. L. Siscoe, V. M. Vasyliunas, C. K. Goertz, and C. M. Yeates, Plasma observations near Jupiter: Initial results from Voyager 1, Science, 204, 987, 1979.

Cloutier, P. A., R. E. Daniell, Jr., A. J. Dessler, and T. W. Hill, A cometary ionosphere model for Io, Astrophys. Space Sci., 55, 93, 1978.

Drell, S. D., H. M. Foley, and M. A. Ruderman, Drag and propulsion of large satellites in the ionosphere: An Alfven propulsion engine in space, J. Geophys. Res., 70, 3131, 1965.

Dungey, J. W., Waves and particles in the magnetosphere, in Physics of the Magnetosphere, edited by R. L. Carovillano, J. F. McClay, and H. R. Radoski, D. Reidel, Hingham, Mass., 1968.

Dungey, J. W., Neutral sheets, in The Magnetospheres of Earth and Jupiter, edited by V. Formisano, D. Reidel, Hingham, Mass., 1975.

Goertz, C. K., and P. A. Deift, Io's interaction with the magnetosphere, Planet. Space Sci., 21, 1399, 1973.

Goldreich, P., and D. Lynden-Bell, Io, a Jovian unipolar inductor, Astrophys. J., 156, 59, 1969.

Gurnett, D. A., Sheath effects and related charged-particle acceleration by Jupiter's satellite Io, Astrophys. J., I75, 535, 1972.

Hill, T. W., Inertial limit on corotation, J. Geophys. Res., 84, 6554, 1979.

Iijima, T., and T. A. Potemra, The amplitude distribution of fieldaligned currents at northern high latitudes observed by Triad, $J$. Geophys. Res., 81, 2165, 1976.

Kennel, C. F., and F. V. Coroniti, Is Jupiter's magnetosphere like a pulsar's or Earth's?, in The Magnetospheres of Earth and Jupiter, edited by V. Formisano, p. 451, D. Reidel, Hingham, Mass., 1975.

Kivelson, M. G., J. A. Slavin, and D. J. Southwood, Magnetospheres of the Galilean satellites, Science, 205, 491, 1979.

Kliore, A. J., G. Fjeldbo, B. L. Seidel, D. N. Sweetnam, T. T. Ses- plaukis, P. M. Woiceshyn, and S. I. Rasool, Atmosphere of Io from Pioneer 10 radio occultation measurements, Icarus, 24, 407, 1975.

Krimigis, S. M., et al., Low-energy charged particle environment at Jupiter: A first look, Science, 204, 998, 1979.

Lin, R. P., R. E. McGuire, H. C. Howe, K. A. Anderson, and J. E. McCoy, Mapping of lunar surface remanent magnetic fields by electron scattering, Proc. Lunar Sci. Conf. 6th, 2971, 1975.

Mallinckrodt, A. J., and C. W. Carlson, Relations between transverse electric fields and field-aligned currents, J. Geophys. Res., 83, 1426, 1978.

Maltsev, Yu. P., S. V. Leontyev, and W. B. Lyatsky, Excitation of Pi-2 pulsations by a flare of aurora, Planet. Space Sci, 22, 1519, 1974.

Marshall, L., and W. F. Libby, Stimulation of Jupiter's radio emission by Io, Nature, 214, 126, 1967.

Mcllwain, C. E., and R. W. Fillius, Differential spectra and phase space densities of trapped electrons at Jupiter, J. Geophys. Res., 80, 1341, 1975.

Mogro-Campero, A., and R. W. Fillius, The absorption of trapped particles by the inner satellites of Jupiter and the radial diffusion coefficient of particle transport, J. Geophys. Res., 81, 1289, 1976.

Ness, N. F., M. H. Acuna, R. P. Lepping, L. F. Burlaga, K. W. Behannon, and F. M. Neubauer, Magnetic field studies at Jupiter by Voyager 1: Preliminary results, Science, 204, 982, 1979.

Neubauer, F. M., Nonlinear standing Alfven wave current system at Io: Theory, J. Geophys. Res., 85, 1171, 1980.

Scarf, F. L., D. A. Gurnett, and W. S. Kurth, Jupiter plasma wave observations: An initial Voyager 1 overview, Science, 204, 991, 1979.

Schatten, K. H., and N. F. Ness, The magnetic-field geometry of Jupiter and its relation to Io-modulated Jovian decametric radio emission, Astrophys. J., 165, 621, 1971.

Shawhan, S. D., Io sheath-accelerated electrons and ions, J. Geophys. Res., 81, 3373, 1976.

Simpson, J. A., D. C. Hamilton, R. B. McKibben, A. Mogro-Campero, K. R. Pyle, and A. J. Tuzzolino, The protons and electrons trapped in the Jovian dipole magnetic field region and their interaction with Io, J. Geophys. Res., 79, 3522, 1974.

Smith, E. J., L. Davis, Jr., D. E. Jones, P. J. Coleman, Jr., D. S. Colburn, P. Dyal, C. P. Sonett, and A. M. A. Frandsen, The planetary magnetic field and magnetosphere of Jupiter: Pioneer $10, J$. Geophys. Res., 79, 3501, 1974.

Stone, E. C., and A. L. Lane, Voyager 1 encounter with the Jovian system, Science, 204, 945, 1979.

Thomsen, M. F., Jovian magnetosphere-satellite interactions: Aspects of energetic charged particle loss, Rev. Geophys. Space Phys., 17, $369,1979$.

Warwick, J. W., et al., Voyager i planetary radio astronomy observations near Jupiter, Science, 204, 995, 1979.

Webster, D. L., A. Y. Alksne, and R. C. Whitten, Does Io's ionosphere influence Jupiter's radio bursts, Astrophys. J., 174, 685, 1969.

(Received December 15, 1979; revised April 8, 1980; accepted April 29, 1980.) 